\title{
BMJ Open Effectiveness of progesterone supplementation in women presenting low progesterone levels on the day of frozen embryo transfer: a randomised controlled trial
}

Thi Minh Chau Le (D) , ${ }^{1}$ Khue Tu Duong, ${ }^{1}$ Quoc Anh Nguyen, ${ }^{1}$ Phuc Thinh Ong, ${ }^{2}$ Thi Hong Nhung Nguyen, ${ }^{1}$ Thi Cam Thu Thai, ${ }^{1}$ Quang Thanh Le,${ }^{3}$ Matheus Roque, ${ }^{4}$ Carlo Alviggi ${ }^{5}$

To cite: Le TMC, Duong KT, Nguyen QA, et al.

Effectiveness of progesterone supplementation in women presenting low progesterone levels on the day of frozen embryo transfer: a randomised controlled trial. BMJ Open 2022;12:e057353. doi:10.1136/ bmjopen-2021-057353

- Prepublication history for this paper is available online. To view these files, please visit the journal online (http://dx.doi. org/10.1136/bmjopen-2021057353).

Received 14 September 2021 Accepted 26 January 2022

Check for updates

(c) Author(s) (or their employer(s)) 2022. Re-use permitted under CC BY-NC. No commercial re-use. See rights and permissions. Published by BMJ.

For numbered affiliations see end of article.

Correspondence to

Dr Thi Minh Chau Le;

dr.lethiminhchau@gmail.com

\section{ABSTRACT}

Introduction Progesterone is an essential hormone involved in the process of implantation and pregnancy maintenance. Evidence from recent studies has supported the importance of serum progesterone level around the time of embryo transfer in hormonal replacement therapy frozen embryo transfer cycles and recommended the need for individualised luteal support. Low progesterone around the time of embryo transfer is found to be associated with decreased rate of pregnancy after frozen embryo transfer. This single-centre, longitudinal, randomised, interventional controlled study aims to compare the rate of ongoing pregnancy between two groups of women with progesterone level below $10 \mathrm{ng} / \mathrm{mL}$ on the day of frozen embryo transfer: the study group using $800 \mathrm{mg}$ vaginal micronised progesterone supplemented with $50 \mathrm{mg}$ intramuscular progesterone per day and the control group using only $800 \mathrm{mg}$ vaginal micronised progesterone. Methods and analysis We enrol patients who are undergoing frozen embryo transfers with blastocyststage or cleavage-stage embryos and who satisfy the inclusion and exclusion criteria. After signing the informed consent, participants are randomised into two groups: the study group using vaginal micronised progesterone supplemented with progesterone intramuscular $50 \mathrm{mg}$ per day and the control group using only vaginal micronised progesterone. Randomisation will be performed using $\mathrm{R}$ software at a 1:1 allocation ratio. Sequentially numbered, opaque sealed envelopes are used for allocation. The primary outcome is the rate of ongoing pregnancy. To demonstrate a difference of $10 \%$ with regard to rate of ongoing pregnancy, at least 370 participants per arm are required (type I error $\alpha=0.05$, power $=0.8$ ). Assuming a dropout rate of $10 \%$, a total of 824 patients (412 per group) will be invited.

Ethics and dissemination This study was approved by the Ethics Committee of Tu Du Hospital on 17 May 2021 (reference number: 1251/QĐ-BVTD). All participants provide informed consent before being enrolled in the study. The results of our study will be submitted to reproductive medicine conferences and journals. Trial registration number NCT04897269.
Strengths and limitations of this study

This is a single-centre, longitudinal, randomised, interventional controlled pharmacological study.

- The intended sample size is large with 824 participants

- Our study is not designed to detect a small difference despite the large sample size.

\section{INTRODUCTION}

Progesterone is an essential hormone in the process of implantation and pregnancy maintenance. Its crucial roles include luteal phase support, modulation of maternal immune response, suppression of inflammatory response, reduction of uterine contractility and improvement of uteroplacental circulation. ${ }^{1}$ Progesterone is a steroid produced primarily by the corpus luteum and the placenta. An investigation conducted by Csapo et $a l^{2}$ showed that luteectomy in early pregnancies induced abortion. Progesterone is necessary to obtain a secretory phase transformation of the endometrium. During the luteal phase, it prepares the endometrium for pregnancy by stimulating proliferation in response to human chorionic gonadotropin (hCG). ${ }^{3}$ During conditions of insufficient progesterone, which is defined as a luteal phase defect, progesterone may not maintain normal secretory endometrium and may prevent normal embryo implantation and growth. ${ }^{4}$ A mid-luteal serum progesterone $<10 \mathrm{ng} / \mathrm{mL}$ was established to predict a luteal phase defect. ${ }^{5}$

Nowadays, frozen-thawed embryo transfer (FET) is being performed worldwide due to several factors, including implementation of 
vitrification with an extremely high survival rate, prevention of ovarian hyperstimulation syndrome and avoiding the negative effect of late follicular elevating progesterone on embryo implantation. ${ }^{6}$ With regard to endometrium preparation in the FET cycles, no protocol is definitely better than the others. ${ }^{78}$ Hormonal replacement therapy (HRT) is a commonly used protocol ${ }^{8}$ due to its flexibility and convenience ${ }^{9}$ in programming the embryo transfer day and excellent results. ${ }^{10}$ In this method, oestrogen is administered from the beginning of the menstrual cycle to inhibit follicle stimulating hormone (FSH) intercycle rise and follicular growth, resulting in the corpus luteum not being formed. Exogenous progesterone administration may begin 2, 3 or 5 days before the scheduled day of transfer depending on the stage of the frozen embryos.

Accumulating evidence has supported the importance of the level of serum progesterone at the time of embryo transfer in HRT FET cycles and recommended the need for individualised luteal support. Labarta $e t a l^{11}$ showed that the rate of ongoing pregnancy was significantly lowered in the group of women with serum progesterone level $<9.2 \mathrm{ng} / \mathrm{mL}$ on the day of embryo transfer compared with women with progesterone level $>9.2 \mathrm{ng} / \mathrm{mL}$. In a retrospective cohort study of Gaggiotti-Marre $e t a l,{ }^{12}$ low serum progesterone level $(\leq 10.64 \mathrm{ng} / \mathrm{mL}) 1$ day before FET was associated with a lower rate of live births following FET of euploid embryos. Similarly, Cédrin-Durnerin et al showed that serum progesterone level $<10 \mathrm{ng} / \mathrm{mL}$ was observed in $37 \%$ of FET cycles and was associated with significantly lower rates of pregnancy ( $34 \%$ vs $48 \%, \mathrm{p}=0.04)$ and live births $(17 \%$ vs $31 \%, \mathrm{p}=0.01) .{ }^{10}$ Thereafter, some studies have assessed the benefit of progesterone supplementation in FET cycles when the progesterone level was below the cut-off. In two recent studies of Yarali et al and Álvarez et $a l^{13}{ }^{14} 25 \mathrm{mg}$ subcutaneous progesterone was added if progesterone level 1 day before embryo transfer was below the cut-off. The rates of ongoing pregnancy were comparable between the progesterone supplementation group and the control group whose progesterone level was above the cut-off. Until now, there have been no randomised control trials assessing the effect of adding progesterone on the day of embryo transfer.

Progesterone is most commonly administered as vaginal suppositories. Vaginal progesterone, with the first uterine pass effect, ${ }^{15}$ is absorbed through the vagina and transmitted primarily to the uterus and therefore achieves higher tissue levels than the oral, subcutaneous or intramuscular route. ${ }^{16}$ Moreover, it is painless, selfadministered and rarely induce allergic reactions. ${ }^{16}$ It is recommended that a low level of serum progesterone after vaginal administration may be rescued by an addition of another route of progesterone. ${ }^{10}$ Most of the recent authors used intramuscular progesterone in their studies. ${ }^{13} 14$

This randomised controlled trial is designed to evaluate the effect of progesterone supplementation in patients with low serum progesterone level on the day of embryo transfer. The primary outcome is the rate of ongoing pregnancy between two groups of women with progesterone level below $10 \mathrm{ng} / \mathrm{mL}$ on the day of frozen embryo transfer: the study group using $800 \mathrm{mg}$ vaginal micronised progesterone supplemented with $50 \mathrm{mg}$ progesterone intramuscular per day and the control group using only $800 \mathrm{mg}$ vaginal micronised progesterone.

\section{MATERIALS AND ANALYSIS}

\section{Design, setting and participants}

This is a single-centre, longitudinal, randomised, interventional controlled pharmacological study enrolling women attending in vitro fertilization (IVF) Tu Du at Tu Du Obstetrics and Gynecology Hospital in Vietnam.

Patients undergoing frozen embryo transfers with blastocyst-stage or cleavage-stage embryos and who satisfy the inclusion and exclusion criteria provide blood samples for progesterone measurement on the day of embryo transfer. Patients with progesterone level below $10 \mathrm{ng} / \mathrm{mL}$ on the day of embryo transfer are randomised into two groups: the study group $(50 \mathrm{mg}$ intramuscular progesterone supplementation and $800 \mathrm{mg}$ vaginal micronised progesterone) and the control group (800 mg vaginal micronised progesterone per day).

The following are the detailed inclusion criteria for participants:

- Patients undergoing Assisted Reproductive Technology (ART) with frozen embryo transfer from 18 to 45 years of age.

- Body mass index (BMI) from $\geq 18.5 \mathrm{~kg} / \mathrm{m}^{2}$ to $22.9 \mathrm{~kg} /$ $\mathrm{m}^{2} .{ }^{17}$

- Endometrial preparation with hormone replacement in frozen embryo transfer cycles with day 3 or day 5 embryos.

The following are the exclusion criteria:

- Oocyte donor cycles.

- Surrogacy.

- In vitro maturation of the oocytes.

- Preimplantation genetic testing.

- Abnormal uterus (fibroid types 0-3 according to the Internation Federation of Gynaecology and Obstetrics (FIGO) classification, endometrial polyp, adenomyosis, congenital malformation).

- Uterine fibroid types 4 and 5 (FIGO classification) $>4 \mathrm{~cm}$.

- History of recurrent implantation failure ( $>2$ times of embryo transfer failure).

- Caesarean scar defect (isthmocoele).

- Endometrial thickness $<7 \mathrm{~mm}$ on the day of embryo transfer.

\section{Recruitment and randomisation}

After completing the IVF profile, patients undergo ovarian stimulation, oocyte retrieval and embryo transfer. The remaining embryos are cryopreserved for future use in frozen embryo transfer (FET) cycle. In the FET cycle, patients come to the clinic on days 1-4 of their period to start endometrial preparation. Embryo transfer occurs 


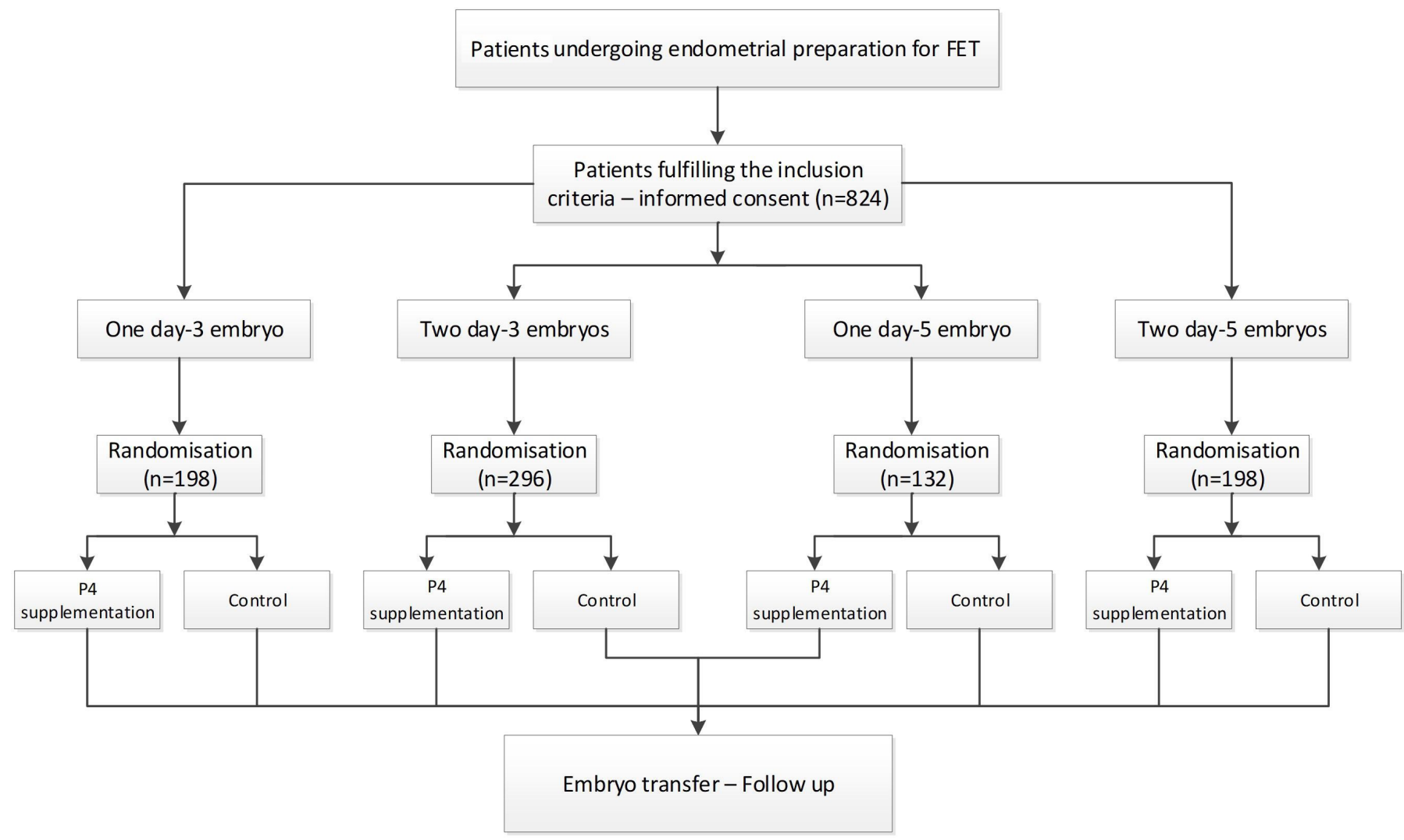

Figure 1 Flow chart of recruitment. FET, frozen-thawed embryo transfer. (P4 - progesterone)

on the third day of progesterone administration for day 3 embryos and on the sixth day for day 5 embryos. On the day of starting progesterone, patients fulfilling the eligibility criteria are invited to participate in the study; the study was explained to all participants. Patients who agree to join sign the informed consent. On the day of embryo transfer, blood is drawn for progesterone measurement. If progesterone level is equal or below $10 \mathrm{ng} / \mathrm{mL}$, patients are randomised to the control group or to the study group.

We employ a stratified randomisation scheme together with permuted block randomisation. Patients are stratified by the number of embryos transferred (one vs two embryos) and the stage of embryos transferred (day 3 vs day 5) (figure 1). Randomisation is then performed using R V.4.0.3 software with package blockrand for each stratum at a 1:1 allocation ratio and with block size determined as 2, 4, 6 and 8 . The randomisation list was prepared by an independent statistician not participating in the recruitment.

Sequentially numbered, opaque sealed envelopes are used for allocation. Envelopes receive numbers in advance and are opened sequentially, only after the participant's name has been written on the appropriate envelope. One member of the research team who labelled the containers is aware of the allocation. Participants and other researchers are unaware of treatment allocation until the envelope is opened. Treatment is not masked to the participants, care providers and investigators, but masked to the outcome assessor.

\section{Intervention and comparator}

Progesterone supplementation group

In this group, patients will be prescribed $50 \mathrm{mg}$ intramuscular progesterone supplementation and $800 \mathrm{mg}$ vaginal micronised progesterone (Cyclogest) per day for 14 days until the day of beta-hCG testing.

\section{Control group}

Patients continue their regular medication $(800 \mathrm{mg}$ vaginal micronised progesterone per day) (Cyclogest) until the day of beta-hCG testing.

If the beta-hCG test is positive, patients will be continued on their prescriptions depending on the study or control group as mentioned above until 7 weeks of pregnancy when the fetal heart is confirmed. This means that in the control group patients are continued on $800 \mathrm{mg}$ vaginal micronised progesterone per day and in the study group patients keep on using $50 \mathrm{mg}$ intramuscular progesterone and $800 \mathrm{mg}$ vaginal micronised progesterone until 7 weeks of pregnancy.

\section{Outcomes}

Primary outcome

- Ongoing pregnancy: ongoing pregnancy is defined as a pregnancy of one or more fetuses with heart beat confirmed by ultrasound at 12 weeks gestation.

\section{Secondary outcome}

- Biochemical pregnancy: a pregnancy diagnosed by detection of beta-hCG in the serum. ${ }^{18}$ 
- Implantation rate: the number of gestational sacs observed divided by the number of embryos transferred (expressed as percentage). ${ }^{18}$

- Miscarriage: spontaneous loss of a clinical pregnancy before 12 completed weeks of gestational age, in which the embryo or the fetus is non-viable and is not spontaneously absorbed or expelled from the uterus. ${ }^{18}$

- Clinical pregnancy: a pregnancy diagnosed by ultrasonographic documentation of at least one fetus with a discernible heart beat. ${ }^{18}$

\section{Endometrial preparation protocol}

After completing the IVF profile, patients undergo ovarian stimulation, oocyte retrieval and embryo transfer. The remaining embryos are cryopreserved for future use in frozen embryo transfer cycle. In the FET cycle, patients come to the clinic on days 1-4 of their period to start endometrial preparation with estradiol valerate. Estradiol valerate is administered at a dosage of $4-8 \mathrm{mg}$ per day. Ultrasound scans are performed every 3-7 days to monitor the development of the endometrium. The dosage of estradiol may be increased up to $16 \mathrm{mg}$ per day to maximise the growth of endometrium. When the endometrium reaches $8 \mathrm{~mm}$ or higher and the duration of endometrial preparation is $\geq 12$ days, vaginal progesterone is started. Embryo transfer occurs on the fourth day of progesterone administration for day 3 embryos and on the sixth day for day 5 embryos.

\section{Sample size estimation}

In a previous study, the rate of ongoing pregnancy of the group with low progesterone level is reported to be $32.7 \% .^{11}$ To demonstrate a difference of $10 \%$ with regard to the rate of ongoing pregnancy, at least 370 participants per arm are required (type I error $\alpha=0.05$, power $=0.8$ ). Assuming a dropout rate of $10 \%$, a total of 824 patients (412 per group) will be invited. We employed a stratified randomisation scheme together with permuted block randomisation. Based on the current distribution of patients' characteristics in the department, participants are stratified by the number of embryos transferred $(40 \%$ for one vs $60 \%$ for two embryos) and the stage of embryo transferred (60\% for day 3 vs $40 \%$ for day 5) into four equal groups:

1. 198 participants with one embryo transferred on day 3 ;

2. 296 participants with two embryos transferred on day 3 ;

3. 132 participants with one embryo transferred on day 5 ; and

4. 198 participants with two embryos transferred on day 5.

\section{Statistical methods}

We will analyse data according to an intention-to-treat principle. To demonstrate baseline variables, continuous data will be presented with mean values and SD or median with IQR according to the normality of data. Categorical data will be described by frequencies and percentages. Comparison of rates of ongoing pregnancy (primary outcome), implantation rates (secondary outcome) and other binary outcomes between the two arms will be performed using $\chi^{2}$ test accompanied by risk ratio and 95\% CI. Quantitative variables will be analysed using Student's t-test or Mann-Whitney test. Multivariable logistic regression will be employed to adjust for potential confounders if the randomisation fails to balance other risk factors between the two arms. All statistical analyses will be performed using R software (Vienna, Austria).

\section{Progesterone measurement}

Serum progesterone level samples are analysed by automated progesterone immunoassays (Cobas 8000). Tu Du Lab is certified by ISO 15189 .

\section{Study duration}

The study started in May 2021, with a recruitment duration of 18-24 months.

\section{Patient and public involvement}

There was no patient and public involvement in the development of the research question, study design or recruitment, and conduct and reporting of the study.

\section{Data monitoring and interim analysis}

Data are monitored, updated and secured by two clinicians and one statistician. The interim analysis will be performed when the first 400 participants have been recruited.

\section{Adverse event monitoring}

Adverse events are closely monitored during the study. On the day of embryo transfer, the participants in the study group are given an instruction leaflet which includes information about side effects, adverse events related to the drug, how to prevent and manage these irritations, and the phone number to call if they feel discomfort. Also, the staffs phone to the patients 2 to 3 times during the luteal support to ask for their compliance to intramuscular progesterone and adverse events.

Common adverse events or adverse reactions related to intramuscular progesterone are pain, swelling at the injection site and breast tenderness. Rare adverse events include severe allergic reactions. All serious adverse events, serious adverse reactions or suspected unexpected serious adverse reactions are recorded and reported to the adverse events system of Tu Du Hospital. When the patient reports an adverse event/adverse reaction (AR) and it is not serious, it is managed as instructions and consultants of the specialists in our clinic. The patient can continue their participation in the study. If the adverse event/adverse reaction is serious, the patient is emergently treated at the Department of Emergency of Tu Du Hospital and is taken out of the study.

Patients can stop participating in the study at any period of time they want. In that case, they are excluded from the study. 


\section{ETHICS AND DISSEMINATION}

Our study was approved by the Ethics Committee of Tu Du Hospital on 17 May 2021 (reference number: 1251/ QĐ-BVTD). All participants provide informed consent before being enrolled in the study. For confidentiality, personal patient information is not shared. The results of our study will be submitted to reproductive medicine conferences and journals.

\section{Author affiliations}

${ }^{1}$ Department of Infertility, Tu Du Hospital, Ho Chi Minh City, Viet Nam

${ }^{2}$ Mathematical Modelling Group, Oxford University Clinical Research Unit, Ho Chi Minh City, Viet Nam

${ }^{3}$ Research Unit, Tu Du Hospital, Ho Chi Minh City, Viet Nam

${ }^{4}$ Department of Reproductive Medicine, Mater Prime, São Paulo, Brazil

${ }^{5}$ Department of Neuroscience, Reproductive Science and Odontostomatology,

University of Naples Federico II, Napoli, Italy

Contributors Study concept and design: TMCL. Draft of the manuscript: TMCL, KTD, QAN, PTO. Critical revision of the manuscript for important intellectual content and study supervision: TMCL, MR and CA. Recruitment: TMCL, KTD, QAN, THNN, TCTT, QTL. Statistical analysis: PTO.

Funding The authors have not declared a specific grant for this research from any funding agency in the public, commercial or not-for-profit sectors.

Competing interests None declared.

Patient and public involvement Patients and/or the public were not involved in the design, or conduct, or reporting, or dissemination plans of this research.

Patient consent for publication Obtained.

Provenance and peer review Not commissioned; externally peer reviewed.

Open access This is an open access article distributed in accordance with the Creative Commons Attribution Non Commercial (CC BY-NC 4.0) license, which permits others to distribute, remix, adapt, build upon this work non-commercially, and license their derivative works on different terms, provided the original work is properly cited, appropriate credit is given, any changes made indicated, and the use is non-commercial. See: http://creativecommons.org/licenses/by-nc/4.0/.

ORCID iD

Thi Minh Chau Le http://orcid.org/0000-0002-7913-900X

\section{REFERENCES}

1 Di Renzo GC, Giardina I, Clerici G, et al. Progesterone in normal and pathological pregnancy. Horm Mol Biol Clin Investig 2016;27:35-48.
2 Csapo Al, Pulkkinen MO, Kaihola HL. The relationship between the timing of luteectomy and the incidence of complete abortions. Am J Obstet Gynecol 1974;118:985-9.

3 Orazov MR, Radzinskiy VE, Nosenko EN, et al. Combination therapeutic options in the treatment of the luteal phase deficiency. Gynecol Endocrinol 2017;33:1-4.

4 Practice Committee of the American Society for Reproductive Medicine. Current clinical irrelevance of luteal phase deficiency: a Committee opinion. Fertil Steril 2015;103:e27-32.

5 Jordan J, Craig K, Clifton DK, et al. Luteal phase defect: the sensitivity and specificity of diagnostic methods in common clinical use. Fertil Steril 1994;62:54-62.

6 Evans J, Hannan NJ, Edgell TA, et al. Fresh versus frozen embryo transfer: backing clinical decisions with scientific and clinical evidence. Hum Reprod Update 2014;20:808-21.

7 Mackens S, Santos-Ribeiro S, van de Vijver A, et al. Frozen embryo transfer: a review on the optimal endometrial preparation and timing. Hum Reprod 2017;32:2234-42.

8 Commissaire M, Cédrin-Durnerin I, Peigné M. [Progesterone and frozen-thawed embryo transfer after hormonal replacement therapy for endometrial preparation: An update on medical practices]. Gynecol Obstet Fertil Senol 2020;48:196-203.

9 Pan Y, Li B, Wang Z, et al. Hormone replacement versus natural cycle protocols of endometrial preparation for frozen embryo transfer. Front Endocrinol 2020;11:546532.

10 Cédrin-Durnerin I, Isnard T, Mahdjoub S, et al. Serum progesterone concentration and live birth rate in frozen-thawed embryo transfers with hormonally prepared endometrium. Reprod Biomed Online 2019;38:472-80.

11 Labarta E, Mariani G, Holtmann N, et al. Low serum progesterone on the day of embryo transfer is associated with a diminished ongoing pregnancy rate in oocyte donation cycles after artificial endometria preparation: a prospective study. Hum Reprod 2017;32:2437-42.

12 Gaggiotti-Marre S, Martinez F, Coll L, et al. Low serum progesterone the day prior to frozen embryo transfer of euploid embryos is associated with significant reduction in live birth rates. Gynecol Endocrinol 2019;35:439-42.

13 Yarali H, Polat M, Mumusoglu S, et al. Subcutaneous luteal phase progesterone rescue rectifies ongoing pregnancy rates in hormone replacement therapy vitrified-warmed blastocyst transfer cycles. Reprod Biomed Online 2021;43:45-51.

14 Álvarez M, Gaggiotti-Marre S, Martínez F, et al. Individualised lutea phase support in artificially prepared frozen embryo transfer cycles based on serum progesterone levels: a prospective cohort study. Hum Reprod 2021;36:1552-60.

15 De Ziegler D, Bulletti C, De Monstier B, et al. The first uterine pass effect. Ann N Y Acad Sci 1997;828:291-9.

16 Zegers-Hochschild F, Schwarze J-E, Borrero C. Delivery routes of progesterone in assisted reproduction. Expert Rev Obstet Gynecol 2006;1:173-82.

17 WHO Expert Consultation. Appropriate body-mass index for Asian populations and its implications for policy and intervention strategies. The Lancet 2004;363:157-63.

18 Zegers-Hochschild F, Adamson GD, Dyer S, et al. The International glossary on infertility and fertility care, 2017. Fertil Steril 2017; 108:393-406. 